\title{
PERFORMANCE STUDIES OF IP OVER ATM VIA ACCESS FLOW CONTROLLED VIRTUAL NETWORKS
}

\author{
L.G. Mason*, F. Vazqez-Abad ${ }^{+}$and B. Kamte* \\ * INRS-Telecommunications, 16 Place du Commerce, Verdun, H3E 1H6, Canada. \\ + University of Montreal, C.P. 6128, Succursale A, Montreal H3C 3J7
}

\begin{abstract}
This paper studies the overlayed virtual network approach to IP/ATM integration which closely resembles the ITU-T Direct approach. Access of IP packets is controlled by a selective windowing mechanism to maximize the network power. New performance results for AAL3/4 and AAL5 protocols are shown. Performance is compared for the Poisson and self-similar IP packet traffic generation processes.
\end{abstract}

\section{Introduction}

The phenomenal growth of the Internet and IP-based applications together with the deployment of an ATM network infrastructure has prompted great interest in suitable schemes for IP/ATM integration. Such an architecture would enable the integration of a high speed Internet service along with native ATM applications while minimizing changes to the widely deployed IP-based applications. A number of proposals for IP/ATM integration have been suggested in the literature, while new schemes continue to appear $[13,15,16]$. The current proposals include classical IP over ATM [8], LAN emulation [5-6], IP-switching [7], Tag switching,[10], CSR (Cell Switch Router) [11], connectionless servers [14], ABR [12], and connectionless virtual networks [1-4].

Our approach, referred to as an overlayed connectionless virtual'network [1-4], employs permanent VCs (VPs) between ATM switches supporting the UBR service with co-located IP routers. This scheme bears a close similarity to the ITU-T standard and indeed preceeded the standard as reported in [14]. This approach scales to large networks, and has advantages over ABR in regard to reliability and complexity, and set-up latency while providing comparable throughput/delay performance and fairness [3-4].

In previous conference publications we have demonstrated how the adaptive isarithmic scheme using the selective windowing mechanism provides an effective procedure for partitioning network bandwidth into virtual networks supporting a real-time connection oriented service class and a delay tolerant connection oriented and/or connectionless class. Reference [24] and references therein provide an overview and details of prior results obtained for the proposed control architecture for integrating IP traffic with other ATM service classes.

The present paper extends this prior work in the following ways, which we believe substantially adds to the fidelity and realism of the simulation results obtained. First we explicitly include connection-less servers at a subset of the ATM switching nodes as proposed in [1] and [14] necessary to provide a scalable architecture. We model the packet access flow control in detail as well as the SAR functions at the CLS for both adaptation layer protocols AAL3/4 and AAL5. Finally we adapted the simulator developed in [23] to generate a self-similar traffic arrival process for IP packets.

\section{Control architecture}

A centralized version of the isarithmic flow control architecture is depicted in Figure 1. This scheme employs adaptive algorithms for the number of permits in circulation and their allocation to source nodes to track the fair-efficient operating point as governed by the maximum of product of powers function. In several publications characteristics of this scheme have been reported for connectionless packet switching networks supporting a homogeneous traffic class [19],[20],[26].

In order to employ the approach for regulating access for connectionless traffic in multi-service ATM networks, an adaptation of the access control referred to as selective window control [22] has been defined and studied by simulation [2-4] for the Indirect approach where VCs are established end-to-end. The specific scheme investigated here, called the aggressive packet selective window (APSW) control, is shown in Figure 2. The decision to admit data traffic is made as soon as there are a positive number of permits at the counter for each incoming IP packet 


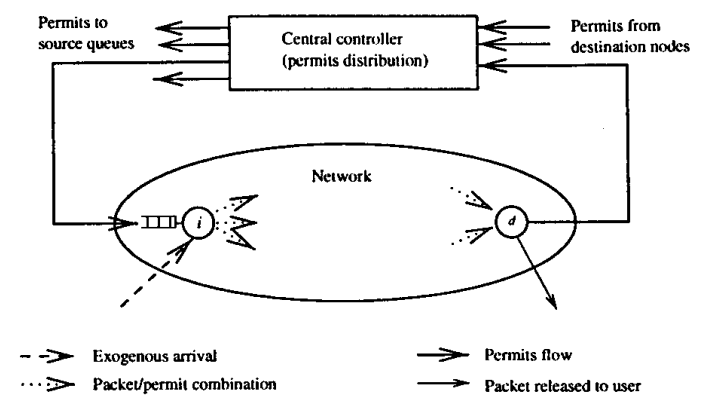

Figure 1: Isarithmic Control Architecture.

and cells of the same packet are granted or denied access all together. The scheme regulates only the data traffic and supposes that the real-time traffic is already controlled by an appropriate preventive control mechanism in order to satisfy its quality of service requirements. With the APSW, if a packet of (say) $n$ cells is accepted in the network, the control grants access to all cells of the packet, irrespective of the value of the counter and subtracts $n$ permits from it, even if the value of the counter becomes negative. Each link in the network is

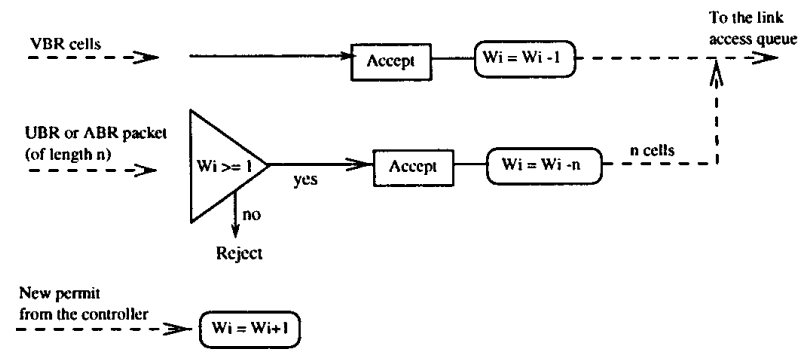

Figure 2: Aggressive Selective Packet Access.

modeled as a server with two queues (see figure 3): one for the real-time cells and the second for the data cells. A non-preemptive priority service is used for real-time traffic to prevent the APSW from having any significant effect on the performance of real-time of traffic. Propagation delay is included in the link model.

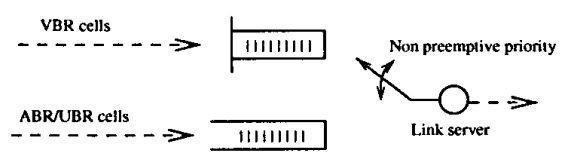

Figure 3: Link Access.

\section{Performance results}

Performance results for a small network shown in Figure 4 have been obtained by Monte-Carlo event simulation. Links are bi-directionnal with equal lengths of $12.2 \mathrm{~km}$ and line speeds of $1.7 \mathrm{Gbits} / \mathrm{sec}$. Characteristics of the IP traffic and the background traffic employed in the simulation studies are defined as follows:

- The VBR traffic is modelled with a two-state MMPP process with parameters $\frac{1}{r_{1}(i, j)}=\frac{1}{r_{2}(i, j)}=4.64 \times 10^{-5}$ seconds, $\lambda_{1(1)}(i, j)=586,200$ cells per second, and $\lambda_{1(2)}(i, j)=92,300$ cells per second.

- The ABR packet arrival process is modelled with a Poisson process with parameter $\lambda_{2}(i, j)=40,400$ packets per second. 


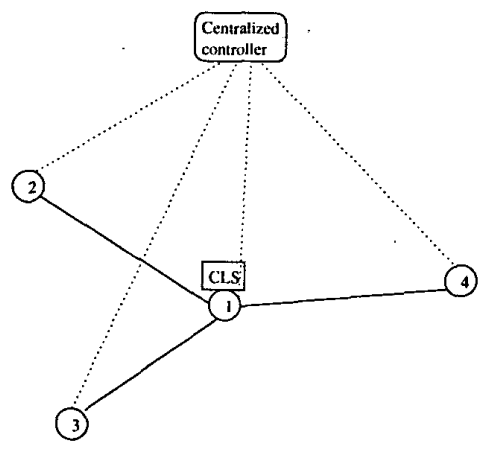

Figure 4: Simulated Network Model

- The UBR packet arrival process is modelled either with a Poisson process with parameter $\lambda_{3}(i, j)=80,800$ packets per second;

OR with the self-similar process obtained by using samples of the fractional ARIMA $(1,0.35,0)$ process [23] as inter-arrival times. Given the sequence of the inter-arrival times, the arrival process is built as the number of arrivals in consecutive time units.

Extensive statistical tests [25] show that the ensuing Hurst parameter for the process is approximatively $H \approx 0.8$ as the one obtained for the measured Internet traffic.

In the results reported here, the probabilities used by the controller for the permits distribution are chosen in proportion to the offered traffic. In [26] it is shown that this allocation is optimal, i.e., it maximizes the product of powers, when the window size is the optimal one. In the adaptive version of the APSW scheme, these probabilities are determined on-line to track the fair-efficient operating point as governed by the maximum of the product of powers function.

Using the mechanism described above, we compared the network performance when the connection-less traffic is modeled with the traditional Poisson process to its performance when the same traffic source is modeled by a self-similar process.

Simulation results (see figure 5) demonstrate that for the same traffic volume the performance for a self-similar traffic source is significantly degraded compared to that for a Poisson source. Interestingly, the optimal window size depends only weakly on the choice of source traffic model.

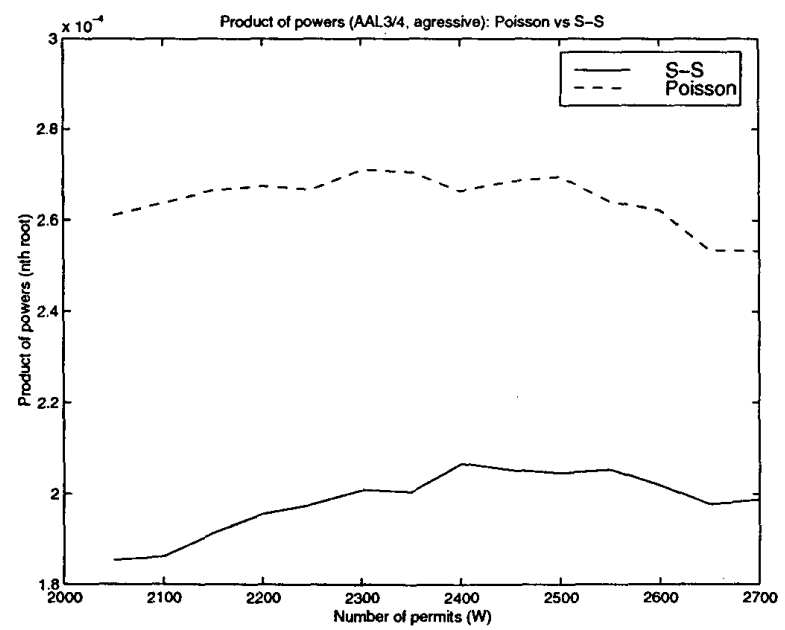

Figure 5: Performance Comparison of Poisson vs. Self-Similar Traffic. 
Two CLS forwarding schemes were compared (under the proposed control): the cell-based forwarding, which uses AAL 3/4 and the packet-based forwarding, which uses AAL 5 [3]. Simulation results, depicted in Figure 6 show that the network performs better when CLS uses AAL3/4 compared to AAL5.

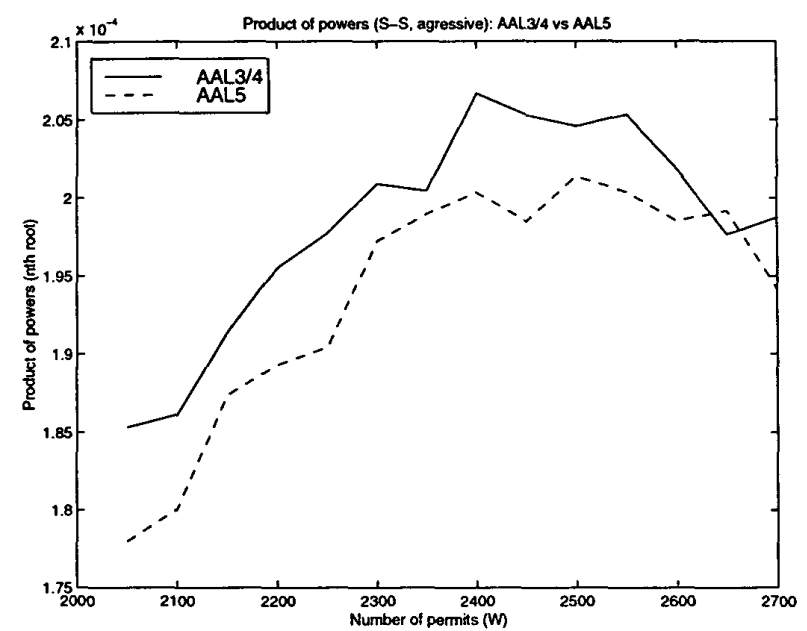

Figure 6: Performance Comparison of AAL3/4 vs. AAL5.

\section{Hierarchical zoning structure for large networks}

To enable the use of the APSW mechanism in large-scale networks, we describe a partially decentralized control architecture structure, which extends the centralized isarithmic approach, by means of hierarchical zoning structure [2]. The results reported here differ from those reported previously, in that the connectionless server, required for scalability, is included. Each zone has its own controller and set of permits and low level zones are connected via gateway nodes to higher level zone. The APSW control is implemented for each zone but there is no access control for cells arriving at the zone's gateway from another zone. Connection-less servers are added at the gateways to support connection-less traffic.

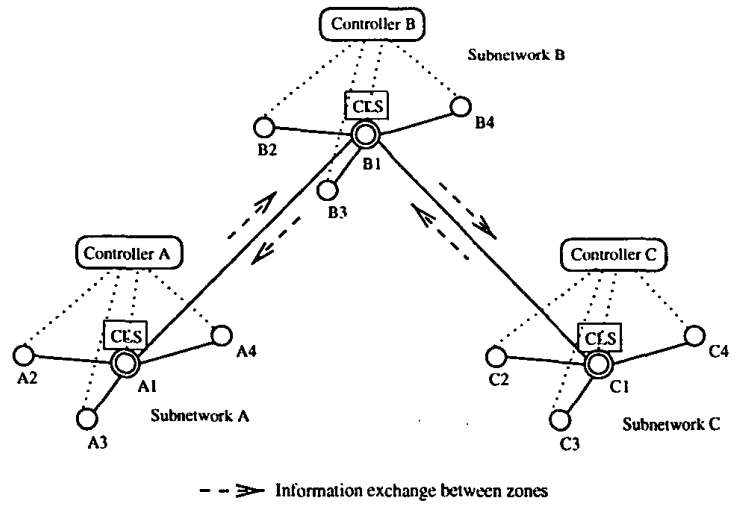

Figure 7: Decentralized Zone Structure.

The computation of the network performance requires the knowledge of the throughputs and delays of all o-d pairs in the network. Each controller needs this information to adjust the number of permits in its zone. As the networks grows in size, the number of measures that must circulate from one zone to the others becomes very large, which will cause an implementation problem. We study the following approximation: rather than carrying all the delay measurements, the management flows carry mean delays only. Each zone computes the arithmitic mean of the delays between its gateway and each node in the zone. Although the mean delay per zone 
is a weighted average of delays per destination within the zone, we have shown under restrictive assumptions that the error in the approximation of the product of powers is minimized by taking instead the arithmitic mean of delays [25]. The management flow then makes this value available to the other zones. This substantially reduces the control overhead associated with the isarithmic scheme and enables it to scale to large networks. Simulation results, shown in Figure 8 , indicate that with this approximation, the global network performance remains almost the same implying that near optimal performance is possible with modest control information overhead.

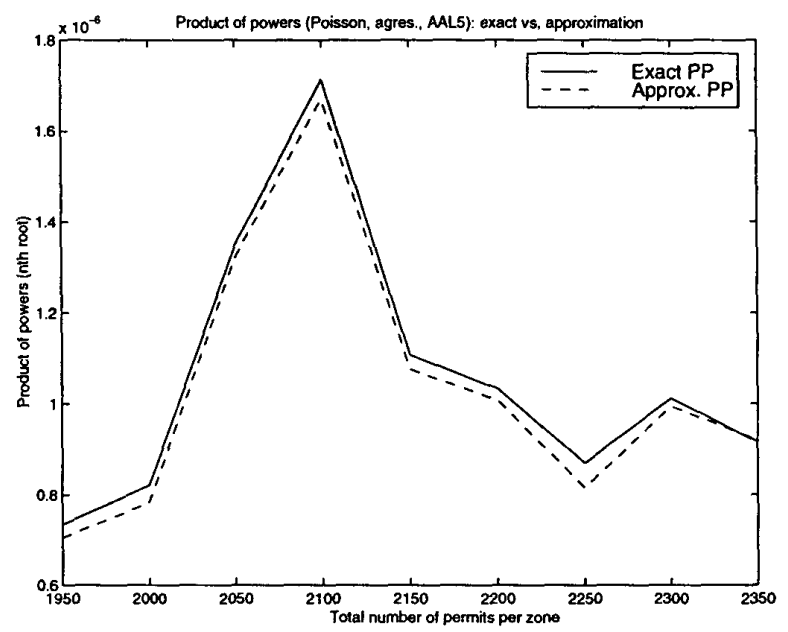

Figure 8: Accuracy of performance approximation

\section{References}

[1] L.G. Mason, Z. Dziong, K-Q Liao, N. Tetreault, "Control Architectures and Procedures for B-ISDN" Proc. of 7th ITC Specialists' Seminar on Broadband Technologies, Morristown New Jersey, Oct.1990

[2] L.G. Mason and A. Pelletier, E. Letourneau, "Decentralized Control Scheme for Large-scale ATM Networks", Broadband Communications' 98, Stuttgart, Germany, April 98, also presented at Workshop on Communicating Informatics and Distributed Systems, Grenoble France, Nov 30-Dec 2, 1994.

[3] Letourneau E. and Mason L.G., "Comparison Study of Credit-Based and Rate-Based ABR Control Scheme" presented at Workshop on ATM Traffic Management, Paris, Dec. 1995.

[4] Letourneau E. , and Mason, L. "Integration Strategies for Flow Control Mechanisms in ATM Networks", ITC Specialist Seminar on Control in Communications, Lund, Sweden, Sept.1996.

[5] ATM Forum. LANE Client Management Specification Version 1, Sept 1995. $\mathrm{ftp}: / / \mathrm{ftp}$.atmforum.com/pub/approved-specs/af-lane-0038.000.ps

[6] ATM Forum. LANE Servers Management Specification Version 1, March 1996.

[7] P. Newman et al. "IP Switching and Gigabit Routers", IEEE Communications Magazine, Jan. 1997.

[8] M. Laubach. "Classical IP and ARP over ATM", Request for Comments, January 1994, RFC1577

[9] G. Parulkar, D. Schmidt and J. Turner, "A Strategy for Integrating IP with ATM", SIGCOMM'95.

[10] Y. Rekhter et al., "Tag Switching Architecture Overview", IETF Internet Draft, Sept. 1996.

[11] H. Esaki et al. "High Speed Datagram Delivery over Internet Using ATM Technology", Proc. Networld+Interop Las Vegas, NV, March 1995, paper E12-1.

[12] F. Bonomi and K.W. Fendick "The Rate-Based Flow Control for ATM Networks", IEEE Network, March/April 1995, pp 25-56. 
[13] P. White, 'ATM Switching and IP Routing Integration: The Next Stage in Internet Evolution", IEEE BSS' 97, Taipei, Dec. 1997.

[14] Brett J. Vickers and Tatsuya Suda, "Connectionless Service for Public ATM Networks" IEEE Communications Magazine, August1994.

[15] Philip Dumortier, "Toward a New IP over ATM Routing Paradigm", IEEE Communications Magazine, January 1998.

[16] Eugenio Guarene, Paolo Fasano, and Vinicio Verellone,"IP and ATM Integration Perspectives". IEEE Communications Magazine, January 1998.

[17] D.W. Davies, "The control of congestion in packet-switching networks", IEEE Trans. On Comm., pp 546$550,1972$.

[18] R. Mazumdar, L. Mason, C. Douligeris, "Fairness in Network Optimized Flow Control: Optimality of Product Forms", IEEE Transactions on Communications May 1991.

[19] M. Cotton, L.G. Mason, "Adaptive Isarithmic Control in Fast Packet-Switching Networks", IEEE Trans. on Communications, Vol 42, 1994.

[20] A. Pelletier, M. Cotton, L.G. Mason, "Combined Adaptive Routing and Flow Control in Fast Packet Switching Networks", Proceedings of COMCON 4, Rhodes, Greece, June 1993

[21] K.-Q. Liao, L.G. Mason, "Self-optimizing Window Flow Control in High Speed Data Networks", Computer Communications, Vol. 16, Number 11, pp. 706-716, 1993.

[22] K.-Q. Liao, L.G. Mason, "A Congestion Control Framework for B-ISDN using Selective Window Control", Proc. of Broadband '94, Paris, March 1994.

[23] Pedro I. Sanchez, "Studies in Traffic Management for ATM Networks", Ph. D. thesis, INRSTelecommunications, Verdun, Quebec, July 1996.

[24] L.G. Mason, F. Vazqez-Abad, B.Kamte, "IP over ATM via Access Flow Controlled Virtual Networks", Proceedings of ICON conference, Ottawa, Canada, July 1998.

[25] Bernard Kamté, "Trafic non connecté sur l'ATM via un contrôle isarithmique de réseaux virtuels", M. Sc. thesis, INRS-Telecommunications, Verdun, Quebec, December 1998.

[26] Felisa J. Vázquez-Abad, Lorne G. Mason, "Adaptive Decentralized Control Under Non-uniqueness of the Optimal Control", Discrete Event Dynamic Systems: theo. and appl. vol. 6, pp 232-359 (1996). 